\title{
Differential diagnosis of solitary pulmonary nodules with dual-source spiral computed tomography
}

\author{
ZHITAO SHI $^{1 *}$, YANHUI WANG ${ }^{1 *}$ and XUEQI HE ${ }^{2}$ \\ ${ }^{1}$ CT Room, Affiliated Hospital of Jining Medical University; ${ }^{2}$ Jining Medical University, \\ Jining, Shandong 272029, P.R. China
}

Received April 28, 2016; Accepted July 15, 2016

DOI: $10.3892 /$ etm.2016.3528

\begin{abstract}
The aim of the present study was to analyze the value of applying dual-source 64-layer spiral computed tomography (CT) in the differential diagnosis of solitary pulmonary nodules (SPNs). Mediastinal windows from 45 cases were selected to study SPNs (maximum diameter, $\leq 3 \mathrm{~cm}$ ), and the pathological nature of lesions was determined by clinical and pathological diagnosis. Conventional 64-layer spiral CT scanning, local enhancement and 3D recombination technologies were used to determine the occurrence rate, lesion diameter, degree of enhancement, lobular sign, spicule sign, pleural indentation sign, vessel convergence sign and bronchus sign. The final diagnoses indicated 34 cases of malignant SPNs (75.6\%) and 11 benign cases $(24.4 \%)$. When the nodule diameter in the malignant group was compared with that of the benign group, the difference was not statistically significant $(\mathrm{P}>0.05)$. Nodules in the malignant group showed inhomogeneous enhancement while nodules in the benign group showed homogeneous enhancement. The enhanced CT values in the malignant group were higher than those in the benign group, and the difference was statistically significant $(\mathrm{P}<0.05)$. The proportion of nodules with lobular sign in the malignant group was significantly higher than that in the benign group $(\mathrm{P}<0.05)$. The proportion of nodules with calcification, vessel convergence sign and bronchus sign in the malignant group were significantly higher than those in the benign group, and the differences were statistically significant $(\mathrm{P}<0.05)$. A comparison of vacuole sign, pleural indentation sign, spiculate protuberance and fat occurrence between the two groups yielded no statistically significant differences $(P>0.05)$. The
\end{abstract}

Correspondence to: Mr. Zhitao Shi, CT Room, Affiliated Hospital of Jining Medical University, 79 Guhuai Road, Jining, Shandong 272029, P.R. China

E-mail: shi_zhitao1@163.com

*Contributed equally

Key words: computed tomography enhancement technique, solitary pulmonary nodule, lobular sign, 3D recombination, spicule sign, pleural indentation sign, calcification sign, vessel convergence sign, bronchus sign sensitivity of CT enhancement was $85.6 \%$, specificity was $79.6 \%$, positive predicated value was $92.3 \%$, and the negative predicted value was $85.2 \%$. In conclusion, SPNs diagnosed by CT enhancement manifested with enhancement degree, lobular sign, calcification, vessel convergence sign and bronchus sign with high diagnostic accuracy.

\section{Introduction}

Solitary pulmonary nodules (SPNs) are solitary and rounded nodular opacities located within the pulmonary parenchyma, not exceeding $3 \mathrm{~cm}$ in diameter (1). SPNs are not associated with pulmonary atelectasis, infection, hilar enlargement or enlarged mediastinal lymph nodes. As the rate of detection of early-stage pulmonary malignant tumors increases, one SPN appears in every 500 chest radiographs (2). SPNs are a common manifestation of multiple benign and malignant diseases and are usually asymptomatic. Qualitative diagnosis of SPNs shows obvious differences in the treatment and prognosis of lung cancer (3). The five-year survival rate of lung cancer is only $10-20 \%$, while that of early-stage lung cancer can reach $70-85 \%$ (4).

At present, the diagnostic techniques for SPNs primarily include fiberoptic bronchoscopy, exfoliative cytology of sputum, chest X-ray, lung needle biopsy, thoracoscopic surgery, ${ }^{18} \mathrm{~F}$-FDG PET/computed tomography (CT) functional imaging, rapid sequence MRI, and dynamic CT perfusion imaging and spectral CT (5). Dynamic CT perfusion imaging has proven effective in the early diagnosis of SPNs. High-resolution CT (HRCT), not only elevates the detection rate of SPNs, but also sufficiently allows for the analysis of internal density of the lesion, edge features, and the association between the lesion and surrounding structures (6).

The aim of the present study was to analyze the value of applying dual-source 64-layer spiral CT enhancement in the differential diagnosis of SPNs.

\section{Patients and methods}

Patients. In total, 45 patients with SPNs were identified using conventional X-ray and CT scanning at the Affiliated Hospital of Jining Medical University (Shandong, China) between January, 2013 and January, 2016. In general, the patients were asymptomatic despite some blood-tinged sputum. All 45 cases 
were verified by pathological or clinical means. The inclusion criteria for the study included: i) Nodule diameter, $\leq 3 \mathrm{~cm}$; ii) nodules had certain sharp edges and could be measured for diameter; iii) presence of other lesions were present within the lung, but nodular morphology was readily distinguishable; and iv) lesion had cavities or calcification. The exclusion criteria for the present study included: i) History of lung injury, surgery or radiation exposure; and ii) definitive history of lung cancer or other cancer types. The study comprised 29 men and 16 women, aged 34-78 years, with an average age of $56.9 \pm 12.6$ years. Twenty-three cases had SPNs in the left lung and 22 in the right. The nodule diameter was $0.5-2.8 \mathrm{~cm}$, and the average diameter was $1.6 \pm 0.7 \mathrm{~cm}$.

The present study was approved by the Ethics Committee of the Affiliated Hospital of Jining Medical University. Informed consent was obtained from patients and their families.

Imaging analysis. A 64-layer spiral CT scanner from GE Medical Systems (Waukesha, WI, USA) was used. Scans were acquired under the following parameters: Voltage $120 \mathrm{kV}$, current $225 \mathrm{~mA}$, rotational speed $0.4 \mathrm{sec} / \mathrm{turn}$, dot spacing 0.531 , FOV $20-24 \mathrm{~cm}$, matrix $512 \times 512$, and $5-\mathrm{mm}$ layer thickness. To capture the images, patients were trained to inspire and hold their breath in a supine position, and the area scanned was from the pleural entrance to the costophrenic angle, including all the pulmonary fields. After the lesions were found, local $0.625-\mathrm{mm}$ thin-layer scanning was conducted and high-resolution algorithms were used to reconstruct the image in combination with dynamic enhanced scanning. Advanced lung analysis software (3D-ALA; GE Healthcare, Piscataway, NJ, USA) on a LightSpeed VCT GE AW 4.4 working station was used to conduct post-analysis processing including 3D-volume reproduction (VR), multiplanar reconstruction, MiniP recombination, and other parameters, as well as store images.

Observation indexes. The following observation indexes were defined as follows: i) Lobular sign: For diagnosis, the arc of the lobular portion was taken as the standard. Lobules with a ratio of chord distance to a length $\geq 0.4$ were classified as a deep lobule, those with a ratio between 0.2 and 0.4 , and those with ratio of $\leq 0.2$ were classified as shallow lobules. Lobules with chord distance of $\leq 4 \mathrm{~mm}$ were defined as thin lobules. Thin lobular sign on VR image was manifested as multiple ball-shaped protuberances on the nodule surface, the protuberance diameter was $n \leq 5.0 \mathrm{~mm}$, and the number of protuberances was $>5$, presenting as a thin nodule-shape. ii) Spicule sign: spicules measuring $<5 \mathrm{~mm}$ were defined as thin and short spicules, and those measuring $>5 \mathrm{~mm}$ were classified as thick and long spicules. iii) Vacuole sign: Non-consolidated alveolar air shadows were visible within lobules. iv) Calcification: CT value was $\leq 200$ HU. v) Pleural indentation sign: The regular linear shadow extracted pleura from the nodule, and the pleural indentation formed a typical horn mouth shape. vi) Spiculate protuberance: Nodule edges protruded sharply with the appearance of a small triangle. vii) Fat: Negative values corresponding to fat density were detected within nodules.

Statistical analysis. Data were analyzed using SPSS 19.0 statistical software. Quantitative data were presented as mean \pm standard deviation. The t-test was used for comparison among groups. Qualitative data were expressed as case number or percentage (\%) and calibration. The $\chi^{2}$ test was used for comparison among groups. The diagnostic value of CT enhancement was calculated according to the formulae: Sensitivity, true positivity/(true positivity + false negativity) case number $x$ 100\%; specificity, true negativity/(true negativity + false positivity) case number x $100 \%$; predicated positive value, true positivity/(true positivity + false positivity) case number x 100\%; and predicted negative value, true negativity/(true negativity + false negativity) case number $x 100 \%$. $\mathrm{P}<0.05$ was considered to indicate statistically significant difference.

\section{Results}

Comparison of nodule diameter and enhancement situation. According to the final diagnoses, there were 34 cases with SPNs (75.6\%), 17 cases of squamous cell lung cancer, 14 cases of glandular cancer and 3 other cases (alveolar cell carcinoma). There were 11 benign cases $(24.4 \%), 7$ cases of cryptogenic organizing pneumonia, 3 cases of tuberculosis and 1 case of hamartoma. When the nodule diameter in the malignant group was compared with the benign group, the difference was not significant $(\mathrm{P}>0.05)$. The nodules in the malignant group demonstrated a mostly inhomogeneous enhancement pattern while those in the benign group showed homogeneous enhancement patterns. The enhanced CT value in the malignant group was higher than that in the benign group, and the difference was statistically significant $(\mathrm{P}<0.05)$ (Table I)

Comparison of lobular and spicule signs. The proportion of nodules with lobular sign was significantly higher in the malignant group compared to the benign group $(\mathrm{P}<0.05)$. The proportions of lobular sign and different lobular sign subtypes in the two groups were compared, and the differences were not statistically significant $(\mathrm{P}>0.05)$ (Table II).

Comparison of other indexes. The proportion of nodules with vessel convergence and bronchus signs in the malignant group were significantly higher than that in the benign group, while calcification was significantly lower than that in the benign group $(\mathrm{P}<0.05)$. Following a comparison of vacuole sign, pleural indentation sign, spiculate protuberance and presence of fat between the two groups, no significant differences were observed between the two groups $(\mathrm{P}>0.05)$ (Table III).

Diagnostic value of CT enhancement. Following calculation according to the abovementioned formulae, the sensitivity was $85.6 \%$, specificity was $79.6 \%$, predicted positive value was $92.3 \%$, and predicted negative value was $85.2 \%$.

\section{Discussion}

Previous evidence showed that the size of lesions shown by CT were basically identical with those after surgery, and correlation coefficients on the $\mathrm{x}-, \mathrm{y}$ - and $\mathrm{z}$-axes were $0.710,0.944$ and 0.875 , respectively (7). SPN diameter may influence the 
Table I. Comparison of nodule diameter and enhancement.

\begin{tabular}{|c|c|c|c|c|c|}
\hline Groups & Case no. & Nodule diameter $(\mathrm{cm})$ & $\begin{array}{c}\text { Homogeneous } \\
\text { enhancement }\end{array}$ & $\begin{array}{c}\text { Inhomogeneous } \\
\text { enhancement }\end{array}$ & $\begin{array}{c}\text { CT enhancement } \\
\text { value }(\mathrm{HU})\end{array}$ \\
\hline Malignant & 34 & $1.5 \pm 0.8$ & $5(14.7)$ & $29(85.3)$ & $142.6 \pm 36.9$ \\
\hline Benign & 11 & $1.6 \pm 0.5$ & $7(63.6)$ & $4(36.4)$ & $105.7 \pm 28.5$ \\
\hline $\mathrm{t}\left(\chi^{2}\right)$-test & & 0.657 & \multicolumn{2}{|r|}{7.827} & 5.629 \\
\hline P-value & & 0.849 & \multicolumn{2}{|r|}{0.005} & 0.033 \\
\hline
\end{tabular}

CT, computed tomography.

Table II. Comparison of lobular sign and spicule sign [case (\%)].

\begin{tabular}{|c|c|c|c|c|c|c|c|c|c|}
\hline Groups & $\begin{array}{c}\text { Case } \\
\text { no. }\end{array}$ & $\begin{array}{l}\text { Lobular } \\
\text { sign }\end{array}$ & $\begin{array}{l}\text { Deep } \\
\text { lobule }\end{array}$ & $\begin{array}{l}\text { Medium } \\
\text { lobule }\end{array}$ & $\begin{array}{c}\text { Shallow } \\
\text { lobule }\end{array}$ & $\begin{array}{c}\text { Thin } \\
\text { lobule }\end{array}$ & $\begin{array}{l}\text { Spicule } \\
\text { sign }\end{array}$ & $\begin{array}{c}\text { Thin and } \\
\text { short spicule }\end{array}$ & $\begin{array}{l}\text { Thick and } \\
\text { long spicule }\end{array}$ \\
\hline Malignant & 34 & $27(79.4)$ & 7 (20.6) & $14(41.2$ & $6(17.6)$ & $8(23.5)$ & $25(73.5)$ & $19(55.9)$ & $6(17.6)$ \\
\hline Benign & 11 & $4(36.4)$ & $1(9.1)$ & $2(18.2)$ & $1(9.1)$ & $1(9.1)$ & $6(54.5)$ & $3(27.3)$ & $3(27.3)$ \\
\hline$\chi^{2}$ & & 5.318 & & & & & 0.652 & \multicolumn{2}{|c|}{0.576} \\
\hline P-value & & 0.021 & & & & & 0.419 & \multicolumn{2}{|c|}{0.448} \\
\hline
\end{tabular}

Table III. Comparison of other indexes [case (\%)].

\begin{tabular}{|c|c|c|c|c|c|c|c|c|}
\hline Groups & $\begin{array}{c}\text { Case } \\
\text { no. }\end{array}$ & $\begin{array}{l}\text { Vacuole } \\
\text { sign }\end{array}$ & Calcification & $\begin{array}{l}\text { Pleural indentation } \\
\text { sign }\end{array}$ & $\begin{array}{c}\text { Spiculate } \\
\text { protuberance }\end{array}$ & Fat & $\begin{array}{c}\text { Vessel convergence } \\
\text { sign }\end{array}$ & $\begin{array}{l}\text { Bronchus } \\
\text { sign }\end{array}$ \\
\hline Malignant & 34 & $15(44.1)$ & $10(29.4)$ & $17(50.0)$ & $10(29.4)$ & $7(20.6)$ & $26(76.5)$ & $28(82.4)$ \\
\hline Benign & 11 & $2(18.2)$ & $8(72.7)$ & $4(36.4)$ & $2(18.2)$ & $3(27.3)$ & $4(36.4)$ & $3(27.3)$ \\
\hline$\chi^{2}$ & & 1.403 & 4.818 & 0.621 & 0.116 & 0.002 & 4.347 & 4.053 \\
\hline $\mathrm{P}$-value & & 0.236 & 0.028 & 0.431 & 0.734 & 0.963 & 0.037 & 0.044 \\
\hline
\end{tabular}

pathological nature of the nodule and corresponding clinical manifestations. As nodule diameter increased, the probability that the nodule was malignant increased significantly. SPN diameter was previously shown to be an independent risk factor for the determination of benignity or malignancy (8). If nodule diameter exceeded $1 \mathrm{~cm}$, this was considered an important value for judgement on the nature of the SPN. However, a small nodule did not exclude the possibility of lung cancer. Fifteen percent of malignant nodules had a diameter of $<1 \mathrm{~cm}$, and $42 \%$ of nodules had a diameter of $<2 \mathrm{~cm}$ (9). At present, CT scanning for SPN growth measures primarily the maximum transverse diameter, maximum area, and volume of nodules. Measurements of the maximum transverse diameter and maximum area are simple and practical to perform, and nodule volume reflected tumor growth more accurately. The 64-row CT LungCare software (Siemens, Erlangen, Germany) is capable of early detection of SPN growth.

A previous study using dynamic enhanced CT for the evaluation of SPNs showed that, the occurrence rate of nodules with lobular sign was $70-100 \%$, and $80-90 \%$ of lobular nodules were malignant lesions. Therefore, the lobular sign was the most common and fundamental sign for the diagnosis of peripheral lung cancer $(10,11)$. The pathogenic mechanism determining the appearance of lobular sign in lung cancer (12) includes: Different degrees of tumor cell differentiation, varying growth speeds at different parts of the tumor edge; the reaction of surrounding tissue, secondary to tumor growth; indentations or incisures generated from tumor growth restriction caused by blood vessels and connective tissues which grew from the inside to the outside of the tumor. The local anatomical structure of lung tissue is composed of connective tissues in the bronchus and blood vessels. In the event of their obstruction, growth of secondary pulmonary lobules is restricted. Due to the volume of small cell lung cancers, they have more shallow lobular signs. HRCT is therefore a more efficient method for observation of shallow lobules of small nodules (13). The observation of deep lobules is also a significant diagnostic sign and its mechanism of formation was shown to be multinuclear tumor cells merging or inhomogeneous growth of different parts of the tumor (14). The outermost edge of a lobule may be the apex of tumor growth, where the tumor-infiltrating neighboring lung tissue was evident, and limited four-indentation at lump edge was formed by obstruction of pulmonary supporting structures such as blood vessel and proliferated fibrous tissues (15). 
Spicule sign was shown to be the main differential diagnostic marker of pulmonary nodules, and the detection rate of lung cancer was approximately $90.0 \%$ (16). The degree of fibrous tissue proliferation within a nodule and surrounding it correlated with the amount of spicules at its edge. Tumor cells gained the capacity for infiltrative growth along alveolar walls, lymphatic vessels, and blood vessels and this was the main factor contributing to the formation of spicule signs (17). The causes of pleural indentation include (18), fibrotic contraction within the tumor body, accompanied or unaccompanied by pleural thickening and adhesion. Tumors can directly invade the pleural edge, and the affected direction of the pleural indentation sign was identical with the direction of bronchial blood vessel bundles of the pulmonary lobe where the lump was located. Whether pleural four-indentation sign was formed was related to lump size and its distance from the pleural plane. Vessel convergence sign represented one or multiple blood vessels infiltrating the tumor body or traversing it, the pulmonary vessel being removed and translocated to the tumor, or blood vessels reaching the tumor edge. Its occurrence rate in peripheral lung cancer was approximately $65.0 \%$ (19). Expanded bronchioles can form an air bronchogram, and the effects of tumors on bronchi include the truncation of the bronchus by a tumor edge, a bronchus being contained by the tumor, and the lumen of the bronchus being pressed and translocated with thickening of the smooth mucous membrane layer of the bronchial wall (20). Other changes include the transmigration stenosis of the lumen and irregular stenosis. Malignant SPNs can cause changes to bronchi including sudden truncation, normality, translation and cone stenosis (21). Calcification is a powerful index in CT for the identification of benign SPNs. Nodule calcification manifests as different forms including center, stratiform, popcorn, needle tip and permeating types. Minor calcifications were found in a small proportion of lung cancers, and the calcified area of the malignant nodules generally did not exceed $10 \%$ of the lesion (22). Fat was not found in any lung cancer or pulmonary sarcoma, only in benign lesions (hamartoma and lipoid pneumonia) (23).

In the present study, when the nodule diameter in the malignant group was compared with that of the benign group, the difference was not statistically significant $(P>0.05)$. Nodules in the malignant group were characterized by inhomogeneous enhancement while nodules in the benign group were characterized by homogeneous enhancement. The enhanced CT value in the malignant group was higher than that in the benign group, and the difference was statistically significant $(\mathrm{P}<0.05)$. When the proportions of lobular sign and its subtypes in the two groups were compared, the differences were not significant $(\mathrm{P}>0.05)$. The proportions of calcification, vessel convergence sign and bronchus sign in the malignant group were significantly higher than those in the benign group $(\mathrm{P}<0.05)$. By contrast, comparisons of vacuole sign, pleural indentation sign, spiculate protuberance and fat occurrence between the two groups were not significantly different $(\mathrm{P}>0.05)$. The sensitivity of $\mathrm{CT}$ enhancement was $85.6 \%$, specificity was $79.6 \%$, predicted positive value was $92.3 \%$ and predicted negative value was $85.2 \%$. In conclusion, CT enhancement mediating the diagnosis of SPNs was mainly manifested at the enhancement degree, lobular sign, calcification, vessel convergence sign and bronchus sign with high diagnostic accuracy.

\section{References}

1. Mets OM, de Jong PA, Scholten ET, Chung K, van Ginneken B, Schaefer-Prokop CM: Subsolid pulmonary nodule morphology and associated patient characteristics in a routine clinical population. Eur Radiol: Jun 2, 2016.

2. Zammit-Maempel I, Kurien R and Paleri V: Outcomes of synchronous pulmonary nodules detected on computed tomography in head and neck cancer patients: 12-year retrospective review of a consecutive cohort. J Laryngol Otol: Mar 15, 2016 (Epub ahead of print).

3. Gómez-Sáez N,Hernández-Aguado I, Vilar J, González-Alvarez I, Lorente MF, Domingo ML, Valero MP, Parker LA and Lumbreras B: Lung cancer risk and cancer-specific mortality in subjects undergoing routine imaging test when stratified with and without identified lung nodule on imaging study. Eur Radiol 25: 3518-3527, 2015.

4. Yung KW, Yung TT, Chung CY, Tong GT, Liu Y, Henderson J, Welbeck D and Oseni S: Principles of cancer staging. Asian Pac J Surg Oncol 1: 1-16, 2015.

5. Mosmann MP, Borba MA, de Macedo FP, Liguori Ade A, Villarim Neto A, de Lima KC: Solitary pulmonary nodule and (18)F-FDG PET/CT. Part 1: epidemiology, morphological evaluation and cancer probability. Radiol Bras 49: 35-42, 2016.

6. Yasaka K, Katsura M, Hanaoka S, Sato J and Ohtomo K: High-resolution CT with new model-based iterative reconstruction with resolution preference algorithm in evaluations of lung nodules: comparison with conventional model-based iterative reconstruction and adaptive statistical iterative reconstruction. Eur J Radiol 85: 599-606, 2016.

7. Perandini S, Soardi GA, Motton M, Augelli R, Zantedeschi L and Montemezzi S: CT imaging features in the characterization of non-growing solid pulmonary nodules in non-smokers. Pol J Radiol 81: 46-50, 2016.

8. Sui X, Meinel FG, Song W, Xu X, Wang Z, Wang Y, Jin Z, Chen J, Vliegenthart $R$ and Schoepf UJ: Detection and size measurements of pulmonary nodules in ultra-low-dose CT with iterative reconstruction compared to low dose CT. Eur J Radiol 85: 564-570, 2016.

9. Kikano GE, Fabien A and Schilz R: Evaluation of the solitary pulmonary nodule. Am Fam Physician 92: 1084-1091, 2015.

10. Cha MJ, Lee KS, Kim HS, Lee SW, Jeong CJ, Kim EY and Lee HY: Improvement in imaging diagnosis technique and modalities for solitary pulmonary nodules: from ground-glass opacity nodules to part-solid and solid nodules. Expert Rev Respir Med 10: 261-278, 2016.

11. Ye XD, Yuan Z, Ye JD, Li HM and Xiao XS: Dynamic enhanced CT evaluation of solitary pulmonary nodules. Zhonghua Zhong Liu Za Zhi 33: 308-312, 2011 (In Chinese).

12. Perandini S, Soardi GA, Motton M, Rossi A, Signorini M and Montemezzi S: Solid pulmonary nodule risk assessment and decision analysis: comparison of four prediction models in 285 cases. Eur Radiol: Dec 8, 2015 (Epub ahead of print).

13. Zhang M, Zhuo N, Guo Z, Zhang X, Liang W, Zhao S and He J: Establishment of a mathematic model for predicting malignancy in solitary pulmonary nodules. J Thorac Dis 7: 1833-1841, 2015.

14. Dziedzic R and Rzyman W: Incidentally diagnosed pulmonary nodule: a diagnostic algorithm. Kardiochir Torakochirurgia Pol 11: 397-403, 2014.

15. Gao F, Ge X, Li M, Zheng X, Xiao L, Zhang G and Hua Y: CT features of lung scar cancer. J Thorac Dis 7: 273-280, 2015.

16. Zhang X, Yan HH, Lin JT, Wu ZH, Liu J, Cao XW and Yang XN: Comparison of three mathematical prediction models in patients with a solitary pulmonary nodule. Chin J Cancer Res 26: 647-652, 2014.

17. Gould MK, Tang T, Liu IL, Lee J, Zheng C, Danforth KN, Kosco AE, Di Fiore JL and Suh DE: Recent trends in the identification of incidental pulmonary nodules. Am J Respir Crit Care Med 192: 1208-1214, 2015.

18. Harzheim D, Eberhardt R, Hoffmann H and Herth FJ: The solitary pulmonary nodule. Respiration 90: 160-172, 2015. 
19. Yankelevitz DF, Yip R, Smith JP, Liang M, Liu Y, Xu DM, Salvatore MM, Wolf AS, Flores RM and Henschke CI; International Early Lung Cancer Action Program Investigators Group: CT screening for lung cancer: nonsolid nodules in baseline and annual repeat rounds. Radiology 277: 555-564, 2015

20. Barbosa FG: PET/CT in the evaluation of pulmonary solitary nodule. Radiol Bras 49: XI, 2016.

21. Zheng B, Zhou X, Chen J, Zheng W, Duan Q and Chen C: A modified model for preoperatively predicting malignancy of solitary pulmonary nodules: an asia cohort study. Ann Thorac Surg 100: 288-294, 2015.
22. Alpert JB, Lowry CM and Ko JP: Imaging the solitary pulmonary nodule. Clin Chest Med 36: 161-178, vii, 2015.

23. Baldwin DR: Prediction of risk of lung cancer in populations and in pulmonary nodules: significant progress to drive changes in paradigms. Lung Cancer 89: 1-3, 2015. 